\title{
Glutamic Acid Decarboxylase cDNA: Nucleotide Sequence Encoding an Enzymatically Active Fusion Protein
}

\author{
Yutaka Kobayashi, Daniel L. Kaufman, and Allan J. Tobin \\ Department of Biology, Molecular Biology Institute, and Brain Research Institute, University of California, Los Angeles, \\ California 90024
}

\begin{abstract}
Glutamic acid decarboxylase (GAD; E.C. 4.1.1.15) catalyzes the production of GABA, the major inhibitory neurotransmitter in the mammalian brain. We recently isolated a lambda gt-11 recombinant, lambda-GAD, that contains the cDNA for GAD from feline brain (Kaufman et al., 1986). Interestingly, the beta-galactosidase-GAD fusion protein encoded by lambda GAD is enzymatically active, catalyzing the conversion of glutamate to $\mathrm{CO}_{2}$ and GABA.
\end{abstract}

Here we report the nucleotide sequence of feline GAD cDNA. It consists of 2265 bases, with a continuous open reading frame of 625 codons. The derived sequence contains the sequence Asn-Pro-His-Lys, which is identical to sequence at the pyridoxal phosphate-binding site of porcine DOPA decarboxylase (Bossa et al., 1977).

The first ATG sequence in the open reading frame begins at nucleotide residue 118 . The 585 codons $3^{\prime}$ to this putative initiation site predict an amino acid composition, $\mathrm{N}$-terminal residue, and molecular size consistent with published characterizations of GAD.

Glutamic acid decarboxylase (GAD; E.C. 4.1.1.15) catalyzes the synthesis of GABA, the major inhibitory neurotransmitter in the brain (Roberts et al., 1976; Hertz et al., 1983). Because of its importance to the normal functioning of the brain and of its suspected involvement in the pathogenesis of seizures and movement disorders, a number of laboratories have undertaken to isolate and study GAD from a number of species (Wu et al., 1973; Blindermann et al., 1978; Oertel et al., 1981; Spink et al., 1985). Although no sequence information for GAD has been published, antisera to purified GAD have been used to identify GABA-producing neurons in the brain (Saito et al., 1974; Mugnaini and Oertel, 1985).

Using one of these antisera (Oertel et al., 1981), we isolated a GAD cDNA clone from a bacterial expression library in the vector lambda gt-11 (Kaufman et al., 1986). This library was made from cDNA copied from the poly(A) RNA of feline occipital cortex.

In lambda gt-11 expression libraries, cDNAs are inserted near the 3 '-terminus of the $F$. coli lac7, gene (Young and Davis, 1983).

Received Sept. 26, 1986; revised Mar. 12, 1987; accepted Mar. 17, 1987

We thank the following people for many helpful discussions: J. Baumer, $\mathbf{M}$. Erlander, G. Frantz, D. Gottlieb, J. Menkes, D. Nierlich, A. Simpson, L. Simpson, N. Tillakaratne, T. Wood, and C. Wuenschell. This work was supported by grants to A.J.T. from NINCDS (NS 22256 and NS 20356) and by a program project grant to Dr. A. V. Delgado-Escueta (PO1 NS 21908). D.L.K. is supported by a USPHS Training Grant in Cell and Molecular Biology (GM 07185).

Correspondence should be addressed to Dr. Allen J. Tobin, Department of Biology, University of California, 405 Hilgard Avenue, Los Angeles, CA 90024.

Copyright (C) 1987 Society for Neuroscience $0270-6474 / 87 / 092768-05 \$ 02.00 / 0$
Infected and induced bacteria produce a fusion protein consisting of the amino terminal 1006 amino acid residues of betagalactosidase followed by the sequence encoded in the cDNA. Recombinant phage containing GAD cDNA directs the synthesis of a fusion protein that reacts with 3 independently derived antisera to GAD (Kaufman et al., 1986). The fusion polypeptide produced by lambda-GAD is enzymatically active: It converts glutamate to stoichiometric amounts of carbon dioxide and GABA. Since no amino acid sequence data are available for GAD from any species, these immunological and functional criteria have established the identity of lambda-GAD. In addition, RNA transcribed from GAD cDNA specifically hybridizes in situ to the RNA of GABA-containing neurons in the cerebellum and other brain regions (Wuenschell et al., 1986, 1987).

Here we report the nucleotide sequence of this GAD cDNA and the corresponding amino acid sequence of feline GAD. Neither the nucleotide sequence nor the derived amino acid sequence of GAD shows a significant similarity to other sequences in the Genbank or NBRF databases. The predicted primary structure contains a sequence of 4 amino acid residucs (Asn-Pro-His-Lys) identical to a sequence in the pyridoxal phosphate-binding site of pig DOPA decarboxylase (Bossa et al., 1977). We suggest that this sequence also serves as the site of pyridoxal phosphate binding in GAD.

\section{Materials and Methods}

Preparation and fragmentation of feline GAD CDNA. To obtain the sequencing templates, we recloned the cDNA insert from lambda-GAD into the Eco RI site of M13mp10. Deletion reactions were performed on the replicative form of M13-GAD by the methods of Hong (1982), Anderson (1981), and Dale et al. (1985). Ligation, transformation, and plating were carried out according to the procedure of Messing (1983). Plaques were picked in $3 \mathrm{ml}$ of LB medium and grown overnight at $37^{\circ} \mathrm{C}$. The phage was precipitated twice by polyethylene glycol, and DNA was isolated by phenol and chloroform extraction, followed by ethanol precipitation. The single-stranded DNA was suspended in $25-50 \mu \mathrm{l}$ of $10 \mathrm{~mm}$ Tris- $\mathrm{HCl}, \mathrm{pH} 7.5,1 \mathrm{~mm}$ EDTA.

Whenever possible, we redigested the phage DNA with an enzyme that recognized sites only within the sequence to be deleted, so that phages with undeleted sequences were linearized and became noninfective. For example, to sequence the region adjacent to a Bgl I site (clone S51), we digested the replicative form of M13-GAD, first partially with $\mathrm{Bgl}$ I and then completely with Hind III. The staggered ends were repaired with Klenow enzyme, and infective phages were reformed by ligation. The partial digestion was needed since $\mathrm{Bgl} \mathrm{I}$ has 2 restriction sites in the cloned DNA, one within the insert and another in the vector. A partial digestion with Bgl I could result, however, in a high background of undeleted phage. To eliminate this problem, we treated the ligated DNA with Sma I (which is part of the polylinker of M13mp10). This linearizes undeleted cDNAs and thereby decreases the transformation efficiency. 


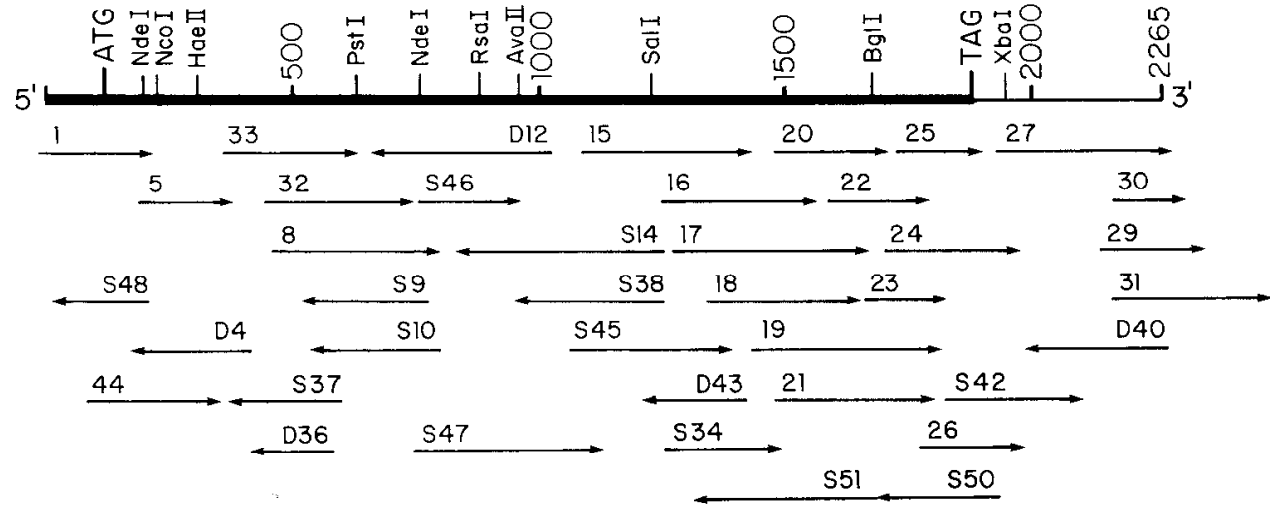

Figure 1. Sequencing strategy for feline GAD cDNA. The open reading frame is shown by the bold line. The position of the putative initiation and termination codons are also shown. The letters and numbers above the arrows refer to the sequenced subclones. The prefix $D$ refers to clones obtained by the procedure of Dale et al. (1985). The prcfix $S$ refers to subclones of restriction fragments. Numbers without an alphabetic prefix refer to clones obtained by the procedure of Hong (1982).
DNA sequencing. DNA was sequenced by the chain-termination method of Sanger et al. (1977) with alpha- ${ }^{35}$ S-dATP (Biggin et al., 1983). Template DNA $(0.5 \mu \mathrm{g})$ was annealed to $2 \mathrm{ng}$ of 17 -nucleotide primer in $8 \mu \mathrm{l}$ of $10 \mathrm{~mm}$ Tris- $\mathrm{HCl}, \mathrm{pH} 8.0,5 \mathrm{mM} \mathrm{MgCl}_{2}$ at $80^{\circ} \mathrm{C}$ for $5 \mathrm{~min}$, followed by $60 \mathrm{~min}$ at room temperature. DNA synthesis was carried out for $30 \mathrm{~min}$ at $37^{\circ} \mathrm{C}$, followed by a 15 min chase. After denaturation at $95^{\circ} \mathrm{C}$ for $3 \mathrm{~min}$ in the presence of formamide, $1 \mu \mathrm{l}$ of the sample was electrophoresed using $6 \%$ acrylamide gel in $7 \mathrm{M}$ urea, $0.1 \mathrm{M}$ Tris, $0.1 \mathrm{M}$ borate, $5 \mathrm{~mm}$ EDTA. The gel was run for 2 or $6 \mathrm{hr}$, fixed in $10 \%$ acetic acid, dried, and autoradiographed on Kodak XAR-5 film overnight at room temperature.

Data processing. Sequence data were analyzed using the program of Staden (1982). Similarity searches employed the program of Wilbur and Lipman (1983).

\section{Results}

The sequencing strategy used is shown in Figure 1. The cloned cDNA is 2265 nucleotides long. Over $99 \%$ of this sequence was determined from both strands. The complete sequence of nucleotides and the inferred sequence of amino acids are shown in Figure 2.

Because the lambda-GAD produces immunoreactive and enzymatically active protein, we knew both the $5^{\prime}-3^{\prime}$ orientation of GAD cDNA in lambda-GAD and its reading frame. The predicted reading frame contains an "open reading frame" 625 codons long, which begins with the sequence GAATTC, corresponding to the Eco $\mathrm{RI}$ site of lambda gt-11, and ends with the TAG stop codon at nucleotide residue 1876 . The first ATG codon begins at nucleotide residue 118 , corresponding to codon no. 40 .

\section{Discussion}

We here report the first data concerning the primary structure of GAD. Previous studies of the structure of GAD have depended on the isolation of a relatively rare and seemingly labile protein. In the 14 years since the first report of purification and characterization, no sequence data have become available ( $\mathrm{Wu}$ et al., 1973). The present data should provide a basis for unraveling many questions concerning the kinetically distinct forms of GAD (Spink et al., 1985).

The 2265 nucleotide residues of feline GAD cDNA represent about $65 \%$ of the total length of GAD mRNA, as determined by RNA blotting experiments (Wood et al., 1986). This feline cDNA forms well-matched hybrids with a 3.7 kbasc mRNA in the brains of cats, mice, rats, and humans, as well as in PC-12 pheochromocytoma cells that have been induced with nerve growth factor (Tillakaratne et al., 1985; Wood et al., 1986). Julien et al. (1987) have recently reported an RNA of about 4 kbase in several regions of rat brain, using an independently derived rat GAD cDNA.
How much of the GAD coding sequence does lambda-GAD contain?

In the absence of amino acid sequence data, we cannot know whether the cloned cDNA contains the entire coding sequence. The following data, however, support the hypothesis that the clone does include the full coding sequence, beginning with the ATG initiation codon at nucleotide residues $118-120$ and ending with the TAG termination codon at residues 1876-1878:

1. The fusion protein encoded by lambda-GAD is enzymatically active. This activity makes it likely that most, if not all, of the GAD coding sequence is contained in the cDNA.

2 . The first codon after the presumed initiation site specifies alanine, which has been reported to be the $\mathrm{N}$-terminal amino acid in both human and rat GAD (Blindermann et al., 1978; Maitre et al., 1978).

3. The open reading frame downstream from the putative initiation codon specifies a polypeptide of $M_{r} 66,000$. Immunoblotting experiments in this and 2 other laboratories, as well as electrophoresis of purified protein under denaturing conditions, indicate that GAD consists of 1 or 2 types of polypeptide chain with $M_{r}$ 59-67,000 (Blindermann et al., 1978; Maitre et al., 1978; Spink et al., 1985; Gottlieb et al., 1986; Kaufman et al., 1986; Julien et al., 1987). Some workers, however, have

Table 1. Comparison of the derived amino acid sequence of feline GAD with the reported compositions of human and rat GAD

\begin{tabular}{lccl} 
& Human & Rat & Feline \\
\hline Asp + Asn & 59 & 52 & 52 \\
Thr & 39 & 26 & 38 \\
Ser & 41 & 37 & 43 \\
Glu + Gln & 69 & 46 & 57 \\
Pro & 17 & 28 & 24 \\
Gly & 45 & 43 & 45 \\
Ala & 47 & 28 & 37 \\
Cys & 10 & 12 & 13 \\
Val & 34 & 37 & 30 \\
Met & 4 & 7 & 17 \\
Ile & 34 & 33 & 31 \\
Leu & 55 & 55 & 56 \\
Tyr & 15 & 13 & 18 \\
Phe & 26 & 20 & 27 \\
Lys & 34 & 43 & 40 \\
His & 6 & 27 & 16 \\
Arg & 28 & 39 & 32 \\
Trp & - & - & 10 \\
\hline
\end{tabular}




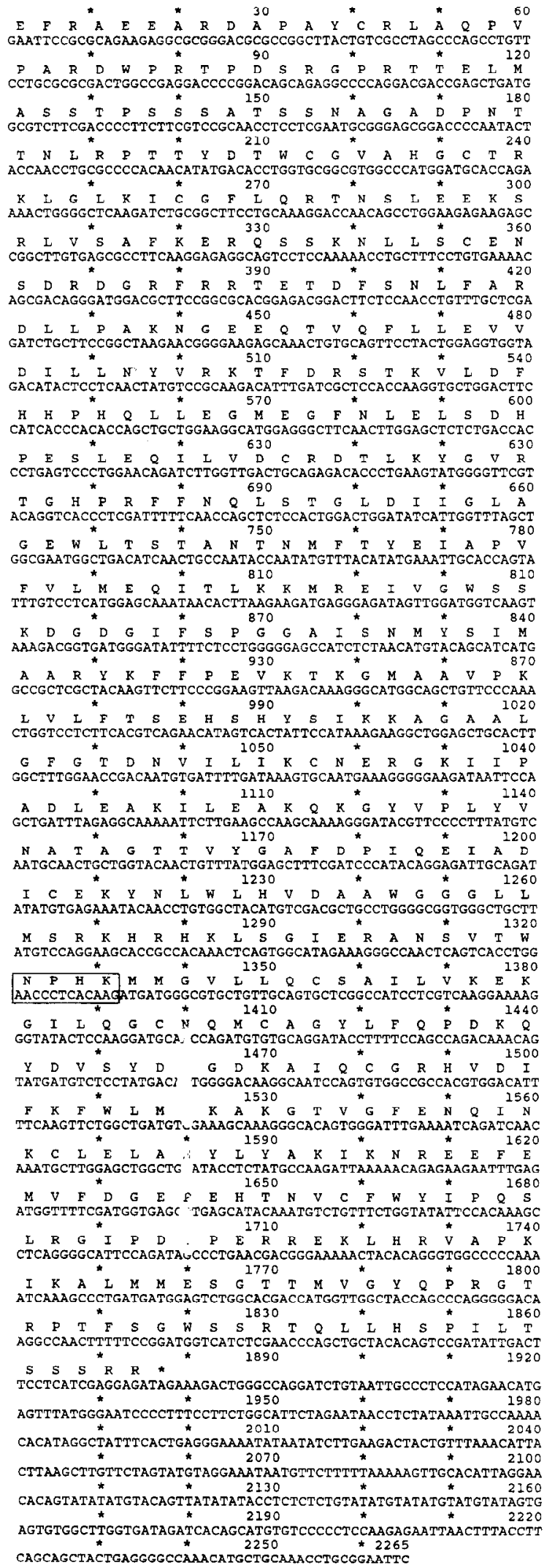

Figure 2. Nucleotide sequence of feline GAD cDNA. The amino acid sequence of the single long open reading frame is shown above the nucleotide sequence. The boxed sequence matches that of the pyridoxal binding site of DOPA decarboxylase from pig kidney (Bossa et al., 1977). proposed subunits of $M_{r} 44,000$ (Wu et al., 1973), 15,000 (Matsuda et al., 1973), and 80,000 and 40,000 (Wu et al., 1986; Denner et al., 1987).

4. The nucleotide sequence contains no additional ATG sequence within 118 residues upstream from the putative initiation site. Another initiation site would therefore specify a polypeptide with a minimum $M$, of 70,000 . But our immunoblotting experiments with feline brain extracts show a maximum $M_{r}$ of 66,000 (Kaufman et al., 1986).

5. The amino acid composition derived from the nucleotide residues 121-1875 closely matches the compositions determined for purified rat and human GAD (Blindermann et al., 1978; Maitre et al., 1978). (See Table 1.)

\section{Similarities of $G A D$ to other proteins}

We have compared the sequence of GAD cDNA with nucleotide sequences in the Genbank DNA sequence database and with the amino acid sequences in the NBRF protein sequence database. We did not find any convincingly similar sequence. Using the dot-matrix program by the University of Wisconsin Genetics Computer Group, we found no significant similarity between GAD and other pyridoxal enzymes either in database or in the recently published sequence of mouse ornithine decarboxylase (Kahana and Nathans, 1985).

When, however, we compared the sequence of feline GAD with the sequences of pyridoxyl peptides for 23 pyridoxal-dependent enzymes (Tanase et al., 1979), we found that the sequence Asn-Pro-His-Lys, encoded by nucleotide residues 13211333 , is identical to the sequence at the pyridoxal binding site of pig DOPA decarboxylase (Bossa et al., 1977). DOPA decarboxylase of Drosophila also contains the same tetrapeptide (Morgan et al., 1986; Eveleth et al., 1986). The homology between feline GAD and Drosophila DOPA decarboxylase is still more extensive: In a 90 amino acid residue sequence in the region of the putative pyridoxal phosphate binding site, 40 are identical in the 2 enzymes.

\section{Other aspects of $G A D$ structure}

Using the algorithm of Kyte and Doolittle (1982), we have found no strongly hydrophobic region that would indicate membrane association. This is consistent with the expectation that GAD is a soluble protein.

\section{An unusual peptide sequence encoded by nucleotide residues $1-117$}

The 39 residues encoded by nucleotide residues $1-117$ are unusually rich, both in charged side chains ( 7 positive and 7 negative groups) and in proline (6). In addition, the codon usage within this scgment diverges from that cxpected for mammals. We are now studying the possible role of this unusual sequence in the unexpected enzymatic activity of the fusion protein.

\section{Possible heterogeneity of GAD molecular forms}

Most studies of mammalian and avian GAD are consistent with the idea of a native $M_{r}$ of $120-140,000$, consisting of 2 identical polypeptides (Blindermann et al., 1978; Maitre et al., 1978; Spink et al., 1985; Gottlieb et al., 1986; Kaufman et al., 1986; Julien et al., 1987). By contrast, Wu et al. (1986) and Denner et al. (1987) say that GAD is a heterodimer consisting of polypeptides with $M_{r}$ of 80,000 and 40,000 .

While it is possible that Wu et al. (1986) and Denner et al. 
(1987) have identified another form of GAD, they do not satisfactorily explain the convergent conclusion that GAD of rats, mice, pigs, humans, and chickens-prepared in 4 other laboratories with different purification protocols-all have subunit $M_{r}$ s of 59-66,000 (Blindermann et al., 1978; Maitre et al., 1978; Spink et al., 1985; Gottlieb et al., 1986; Julien et al., 1987). Nor do they discuss our published comparison of the sizes of immunoreactive GAD polypeptides (Kaufman et al., 1986). That study employed 3 different antibodies: the sheep anti-rat GAD of Oertel et al. (1981); the sheep anti-pig GAD of Spink et al. (1985); and the rabbit anti-mouse GAD of Saito et al. (1974).

Gottlieb et al. (1986) have also inferred a subunit $M_{r}$ of 59,000 for both rat and chicken GAD from immunoblotting studies with a monoclonal antibody to chicken GAD. Immunocytochemical localization of GAD performed with the monoclonal antibody of Gottlieb et al. (1986) and with the polyclonal antibodies of Oertel et al. (1981) and of Saito et al. (1974) all revealed a distribution of immunoreactivity within the mammalian brain consistent with the expected distribution of GAD as inferred from electrophysiology, enzymatic activity, and studies of GABA distribution and uptake (see also Roberts et al., 1976; Hertz et al., 1983; Mugnaini and Oertel, 1985; Wuenschell et al., 1986, 1987).

We and others have studied the cellular distribution of GAD mRNA in the brain, using either RNA transcribed from the cDNA whose sequence we report here (Wuenschell et al., 1986, 1987; Chesselet et al., 1987) or labeled DNA from the rat GAD cDNA of Julien et al. (1987). The inferred distribution of GAD mRNA is that expected from the results of immunocytochemistry, electrophysiology, and GABA uptake (Roberts et al., 1976; Hertz et al., 1983). All these data, together with the GAD enzymatic activity of both the feline and the rat fusion proteins, support the identification of the present clone as GAD. The sequence data that we present here for feline GAD will make it possible to design experiments to define further the identities and enzymatic roles of reported multiple forms of GAD.

\section{References}

Anderson, S. (1981) Shotgun DNA sequencing using cloned DNase I-generated fragments. Nucl. Acids Res. 9: 3015-3027.

Biggin, M. D., T. J. Gibson, and G. F. Hong (1983) Buffer gradient gels and ${ }^{35} \mathrm{~S}$ label as an aid to rapid DNA sequence determination. Proc. Natl. Acad. Sci. USA 80: 3963-3965.

Blindermann, J.-M., M. Maitre, L. Ossola, and P. Mandel (1978) Purification and some properties of L-glutamate decarboxylase from human brain. Eur. J. Biochem. 86: 143-152.

Bossa, F., F. Martini, D. Barra, C. B. Boltattorni, A. Minelli, and C. Turano (1977) The chymotryptic phosphopyridoxyl peptide of DOPA decarboxylase from pig kidney. Biochem. Biophys. Res. Commun. 78: $177-184$.

Chesselet, M.-F., L. Weiss, C. W. Wuenschell, A. J. Tobin, and H.-U. Affolter (1987) Comparative distribution of mRNAs for glutamic acid decarboxylase, tyrosine hydroxylase, and tachykinins in the basal ganglia: An in situ hybridization study in the rodent brain. J. Comp. Neurol. (in press).

Dale, R. M. K., B. A. McClure, and J. P. Houchins (1985) A rapid singlc-stranded cloning strategy for producing a sequential series of overlapping clones for use in DNA sequencing: Application to sequencing the corn mitochondrial $18 \mathrm{~S}$ rDNA. Plasmid 13: 31-40.

Denner, L. A., S. C. Wei, H. S. Lin, C.-T. Lin, and J.-Y. Wu (1987) Brain L-glutamate decarboxylase: Purification and subunit structure. Proc. Natl. Acad. Sci. USA 84: 668-672.

Eveleth, D. D., R. D. Gietz, C. A. Spencer, F. E. Nargang, R. B. Hodgetts, and J. L. Marsh (1986) Sequence and structure of the dopa decarboxylase gene of Drosophila: Evidence for novel RNA splicing variants. EMBO J. 5: 2663-2672.
Gottlieb, D. I., Y.-C. Chang, and J. E. Schwob (1986) Monoclonal antibodies to glutamic acid decarboxylase. Proc. Natl. Acad. Sci. USA 83: 8808-8812.

Hertz, L., E. Kvamme, E. G. McGeer, and A. Schousboe, eds. (1983) Glutamine, Glutamate and GABA in the Central Nervous System, Liss, New York.

Hong, G. F. (1982) A systematic DNA sequencing strategy. J. Mol. Biol. 158: 539-549.

Julien, J.-F., F. Legay, S. Dumas, M. Tappaz, and J. Mallet (1987) Molecular cloning, expression and in situ hybridization of rat brain glutamic acid decarboxylase messenger RNA. Neurosci. Lett. 73:173180.

Kahana, C., and D. Nathans (1985) Nucleotide sequence of murine ornithine decarboxylase mRNA. Proc. Natl. Acad. Sci. USA 82: 16731677.

Kaufman, D. L., J. F. McGinnis, N. R. Krieger, and A. J. Tobin (1986) Brain glutamate decarboxylase cloned in lambda-gt-11: Fusion protein produces gamma-aminobutyric acid. Science 232: 1138-1140.

Kyte, J., and R. F. Doolittle (1982) A simple method for displaying the hydropathic character of a protein. J. Mol. Biol. 157: 105-132.

Maitre, M., J.-M. Blindermann, L. Ossola, and P. Mandel (1978) Comparison of the structures of L-glutamate decarboxylases from human and rat brains. Biochem. Biophys. Res. Commun. 85: 885-890.

Matsuda, T., J.-Y. Wu, and E. Roberts (1973) Electrophoresis of glutamic acid decarboxylase (EC 4.1.1.15) from mouse brain in sodium dodecyl sulphate polyacrylamide gels. J. Neurochem. 21: 167-172.

Messing, J. (1983) New M13 vectors for cloning genes. Methods Enzymol. 101: 20-78.

Morgan, B. A., W. A. Johnson, and J. Hirsh (1986) Regulated splicing produces different forms of dopa decarboxylase in the central nervous system and hypoderm of Drosophila melanogaster. EMBO J. 5:33353342.

Mugnaini, E., and W. H. Oertel (1985) An atlas of the distribution of GABAergic neurons and terminals in the rat CNS as revealed by GAD immunohistochemistry. In Handbook of Chemical Neuroanato$m y$, Vol. 4, A. Björklund and T. Hökfelt, eds., pp. 436-608, Elsevier, Amsterdam.

Oertel, W. H., D. E. Schmechel, M. L. Tappaz, and I. J. Kopin (1981) Production of a specific antiserum to rat brain glutamic acid decarboxylasc by injection of an antigen-antibody complex. Neuroscience 6: $2689-2700$.

Roberts, E., T. N. Chase, and D. B. Tower, eds. (1976) GABA in Nervous System Function, Raven, New York.

Saito, K., R. Barber, J.-Y. Wu, T. Matsuda, E. Roberts, and J. E. Vaughn (1974) Immunohistochemical localization of glutamic acid decarboxylase in rat cerebellum. Proc. Natl. Acad. Sci. USA 71: 269-273.

Sanger, F., S. Nicklen, and A. R. Coulson (1977) DNA sequencing with chain-terminating inhibitors. Proc. Natl. Acad. Sci. USA 74: 5463-5467.

Spink, D. C., T. G. Porter, S. J. Wu, and D. L. Martin (1985) Characterization of three kinetically distinct forms of glutamate decarboxylase from pig brain. Biochem. J. 231: 695-703.

Staden, R. (1982) Automation of the computer handling of gel reading data produced by the shotgun method of DNA sequencing. Nucleic Acids Res. 10: 4731-4751.

Tanase, S., H. Kojima, and Y. Morino (1979) Pyridoxal 5'-phosphate binding site of pig heart alanine aminotransferase. Biochemistry 18 : 3002-3007.

Tillakaratne, N. J. K., S. L. Huttner, and A. J. Tobin (1985) NGF induction of glutamate decarboxylase (GAD) mRNA in pheochromocytoma cell lines. Soc. Neurosci. Abstr. 10: 357.

Wilbur, W. J., and D. J. Lipman (1983) Rapid similarity searches of nucleic acid and protein data banks. Proc. Natl. Acad. Sci. USA 80: 726-730.

Wood, T. L., G. D. Frantz, J. H. Menkes, and A. J. Tobin (1986) Regional distribution of messenger RNAs in postmortem human brain. J. Neurosci. Res. 16: 311-324.

Wu, J.-Y., T. Matsuda, and E. Roberts (1973) Purification and characterization of glutamate decarboxylase from mouse brain. J. Biol. Chem. 248: 3029-3034.

Wu, J.-Y., L. A. Denner, S. C. Wei, C. T. Lin, H. S. Lin, J. W. Liu, and Y. F. Xu (1986) Production and characterization of polyclonal and monoclonal antibodies to rat brain L-glutamate decarboxylase. Brain Res. 373: 1-14. 
Wuenschell, C. W., R. S. Fisher, D. L. Kaufman, and A. J. Tobin (1986) In situ hybridization to localize mRNA encoding the neurotransmitter biosynthetic enzyme glutamate decarboxylase (GAD) in mouse cerebellum. Proc. Natl. Acad. Sci. USA 83: 6193-6197.

Wuenschell, C. W., R. S. Fisher, N. J. K. Tillakaratne, and A. J. Tobin
(1986) In situ detection of GAD mRNA in mouse brain. In In Situ Hybridization in Brain, G. Uhl, ed., pp. 135-149, Plenum, New York. Young, R. A., and R. W. Davis (1983) Yeast RNA polymerase II genes: Isolation with antibody probes. Science $222: 778-782$. 